\title{
Which children with nephrotic syndrome benefit from cyclosporin A or cyclophosphamide?
}

\author{
Malcolm G. Coulthard
}

Received: 3 December 2008 / Accepted: 17 December 2008 /Published online: 3 February 2009

(C) IPNA 2009

Sirs,

I have read with great interest the article from Plank et al. [1]. It is a shame that Plank et al. terminated their excellent randomised controlled trial of the efficacy and safety of cyclosporin A versus pulsed cyclophosphamide in children with steroid-resistant nephrotic syndrome by using the particular statistical protocol that they did [1], as this may have prevented them from obtaining even more important information. Their predetermined plan to cease recruitment if either limb of their study showed overall superior efficacy with a probability of $<0.05$ meant that they did not look separately at the probability values for children with focal segmental glomerulosclerosis (FSGS), and for those who had minimal change or mesangial proliferative histology.

Thus, the study was stopped when nine of the 15 children had fully or partially responded to cyclosporin A, compared to three of the 17 children on cyclophosphamide ( $p=0.03$, Fishers exact test). Setting the threshold for discontinuing the study on reaching a probability of $\leq 0.05$ is not particularly robust. In this instance, the statistical power hinges on the outcome of a single child; if just one more had responded to cyclophosphamide or just one less had responded to cyclosporin A, the probabilities of a null hypothesis would have approximately doubled to $\geq 0.06$.

M. G. Coulthard $(\bowtie)$

Department of Paediatric Nephrology, Royal Victoria Infirmary, Newcastle NE1 4LP, UK

e-mail: Malcolm.Coulthard@nuth.nhs.uk
If the children are analysed by histological groups, the apparent advantage of cyclosporin A over cyclophosphamide seems to be confined to those with FSGS, with no difference observed between the two treatments for children with minimal change or mesangial proliferation. Four of eight children with FSGS responded to cyclosporin A compared to just one of the 13 on cyclophosphamide $(p=0.05)$, whereas among the other children, similar proportions responded to cyclosporin A and cyclophosphamide ( $5 / 7$ vs. $2 / 4 ; p=0.6)$. If these authors had continued recruiting until children in either of the histological groups had shown a robust significant treatment advantage, a highly significant result may have been obtained without the study size needing to be vastly larger. For example, if twice as many children had been recruited and had shown the same trends, then the apparent advantage of cyclosporin A over cyclophosphamide in children with FSGS would have been supported by a $p$ value $=0.003$, while the results for the remainder would have remained insignificant. Perhaps this excellent research group would consider extending their study in this way, even now.

\section{References}

1. Plank C, Kalb V, Hinkes B, Hildebrandt F, Gefeller O, Rascher W, Arbeitsgemeinschaft für Pädiatrische Nephrologie (2008) Cyclosporin A is superior to cyclophosphamide in children with steroidresistant nephrotic syndrome- - a randomized controlled multicentre trial by the Arbeitsgemeinschaft für Pädiatrische Nephrologie. Pediatr Nephrol 23:1483-1493 